\title{
Early response to eptinezumab indicates high likelihood of continued response in patients with chronic migraine
}

\author{
Dawn C. Buse ${ }^{1,2}$, Paul K. Winner ${ }^{3}$, Larry Charleston IV ${ }^{4}$, Joe Hirman ${ }^{5}$, Roger Cady ${ }^{6 *}$ and Thomas Brevig ${ }^{7}$
}

\begin{abstract}
Background: A clinical ability to describe the response trajectory of patients receiving preventive migraine treatment could expedite and improve therapeutic management decisions. This post hoc analysis of the PROMISE-2 study evaluated the consistency and predictive power of Month 1 treatment response on later response in patients with chronic migraine.

Methods: PROMISE-2 was a double-blind, placebo-controlled trial that randomized adults with chronic migraine to eptinezumab $100 \mathrm{mg}, 300 \mathrm{mg}$, or placebo administered IV every 12 weeks for up to 24 weeks (2 infusions over 6 study months). Migraine responder rates (MRRs) were calculated from monthly migraine days over 4-week intervals compared with baseline. Patients were grouped by MRR during Month $1(<25 \%, 25-<50 \%, 50-<75 \%$, and $\geq 75 \%)$, with the number of subsequent study months (Months 2-6) with $\geq 50 \%$ and $\geq 75 \%$ MRR calculated in each subgroup. A similar analysis was conducted using Patient Global Impression of Change (PGIC) rating to define Month 1 subgroups (very much improved, much improved, minimally improved, and no change/worse) and rates of very much improved or much improved PGIC during Months 2-6.
\end{abstract}

Results: In the eptinezumab 100 mg, 300 mg, and placebo groups, respectively, 194/356 (54.5\%), 212/350 (60.6\%), and 132/366 (36.1\%) patients were $\geq 50 \%$ migraine responders during Month 1. More eptinezumab-treated patients were $\geq 75 \%$ migraine responders (100 mg, 110/356 [30.9\%]; 300 mg, 129/350 [36.9\%]; placebo, 57/366 [15.6\%]) and more placebo-treated patients were $<25 \%$ migraine responders (eptinezumab 100 mg, 103/356 [28.9\%]; 300 mg, 80/350 [22.9\%]; placebo, 153/366 [41.8\%]). Among patients who achieved $\geq 75 \%$ migraine response in Month 1, more than one-third attained $\geq 75 \%$ migraine response for all 5 subsequent study months and more than twothirds achieved $\geq 75 \%$ migraine response for $\geq 3$ months. More than two-thirds of those in the very much improved (PGIC) subgroup at Month 1 were much or very much improved for all 5 subsequent months.

Conclusions: In this post hoc analysis of data from PROMISE-2, more eptinezumab-treated than placebo-treated patients were early (Month 1) responders, and most early responders went on to achieve a high level of response for at least half of the 24-week treatment period. Potential for later response in early non-responders was also observed.

Trial registration: ClinicalTrials.gov identifier: NCT02974153; registered November 23, 2016.

Keywords: Eptinezumab, Chronic migraine, Migraine prevention, Clinical response

\footnotetext{
* Correspondence: rcady@rkconsults.com

${ }^{6}$ Lundbeck LLC, IL, Deerfield, USA

Full list of author information is available at the end of the article
}

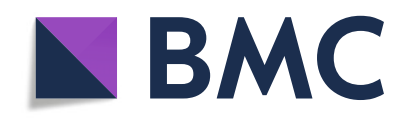

(- The Author(s). 2022 Open Access This article is licensed under a Creative Commons Attribution 4.0 International License, which permits use, sharing, adaptation, distribution and reproduction in any medium or format, as long as you give appropriate credit to the original author(s) and the source, provide a link to the Creative Commons licence, and indicate if changes were made. The images or other third party material in this article are included in the article's Creative Commons licence, unless indicated otherwise in a credit line to the material. If material is not included in the article's Creative Commons licence and your intended use is not permitted by statutory regulation or exceeds the permitted use, you will need to obtain permission directly from the copyright holder. To view a copy of this licence, visit http://creativecommons.org/licenses/by/4.0/. The Creative Commons Public Domain Dedication waiver (http://creativecommons.org/publicdomain/zero/1.0/) applies to the data made available in this article, unless otherwise stated in a credit line to the data. 


\section{Introduction}

For patients living with chronic migraine [1], preventive treatment that effectively reduces the number and burden of migraine attacks has the potential to change lives and daily functioning. By reducing the number of migraine days and associated disability, potentially lessening the likelihood of developing comorbidities, and decreasing the direct and indirect costs associated with chronic migraine $[1,2]$, preventive agents can considerably reduce the burden of this disorder.

The American Headache Society recommends that clinicians help patients establish realistic expectations regarding the anticipated benefits of prescribed preventive migraine medications [3]. However, the recent introduction of calcitonin gene-related peptide (CGRP) antagonists has changed the treatment paradigm for patients requiring migraine prevention. Treatments targeting CGRP typically have a more rapid onset of action [4-18] with no or minimal interim period between treatment initiation and full onset of efficacy, and these attributes have the potential to decisively change patient management. Thus, the ability of clinicians to describe the response trajectory of a particular preventive treatment agent at an early stage could accelerate management decisions related to continuation of that specific treatment, and interim use of acute medication.

Eptinezumab (Vyepti ${ }^{\text {ix }}$, Lundbeck Seattle BioPharmaceuticals, Inc., Bothell, WA, USA), is an intravenously administered monoclonal antibody targeting the CGRP ligand and is approved for the preventive treatment of migraine in adults [19]. In the pivotal PROMISE-2 study of patients with chronic migraine, eptinezumab-at the approved doses of $100 \mathrm{mg}$ and $300 \mathrm{mg}$ - significantly reduced migraine frequency, was well tolerated, and had an acceptable safety profile [16]. The onset of response was rapid, with a $>50 \%$ reduction in migraine prevalence on the day after dosing in the eptinezumab treatment groups. During the first month (Weeks 1-4) of the study, approximately a third of eptinezumab-treated patients had $\mathrm{a} \geq 75 \%$ migraine response [16]. In addition, $45 \%$ and $59 \%$ of patients receiving eptinezumab $100 \mathrm{mg}$ and $300 \mathrm{mg}$, respectively, reported "much" or "very much" improvement through Weeks 1-4, based on the Patient Global Impression of Change (PGIC) [20].

The objective of this post hoc analysis of the PROMISE2 study was to evaluate the general consistency and predictive power of treatment response-defined by reduction in the frequency of monthly migraine days (MMDs) and by patient perception of change in disease during the first month of eptinezumab treatment-on later response outcomes in patients with chronic migraine.

\section{Methods}

\section{Study design, patients, and interventions}

PROMISE-2 (NCT02974153) was a randomized, doubleblind, placebo-controlled, parallel-group, phase 3 clinical trial that evaluated the preventive efficacy, tolerability, and safety of eptinezumab in adults with chronic migraine [16]. The study was conducted in accordance with the International Conference on Harmonisation Good Clinical Practice guidelines, the principles of the Declaration of Helsinki, and all applicable local regulatory requirements. The study was approved by the independent ethics committee or institutional review board for each study site.

Detailed methodology has been published [16]. In brief, eligibility criteria were an age of $18-65$ years, a diagnosis of migraine at or before age 50 years, a history of chronic migraine for at least 12 months before screening, completion of a headache electronic diary (eDiary) on at least 24 of the 28 days during the screening period, and experiencing 15-26 headache days and at least 8 migraine days during the screening period. Key exclusion criteria were presence of a confounding pain disorder or clinically significant pain syndromes, and history or diagnosis of a headache or migraine disorder that did not meet Section 1.3 criteria for chronic migraine per the 2013 International Classification of Headache Disorders, 3rd edition, beta version (ICHD-3 $\beta$ ) [21]. This diagnoses was also made by ICHD beta criteria. This may not be an essential correction as ICHD-3 criteria were not published until 1, 2018. All patients enrolled in the study provided written informed consent before their participation.

Eligible patients were randomized to intravenous eptinezumab $100 \mathrm{mg}$, eptinezumab $300 \mathrm{mg}$, or placebo at 28-30 days after the screening visit and were administered the first dose of study drug on Day 0 , within 8 days after the randomization visit. A second dose was administered on Day 84 (Week 12), with 24 weeks included in the study treatment period. Patients completed a daily eDiary from the time of screening through Week 24 to capture daily headache episodes and migraine attacks (defined per ICHD-3 3 ).

\section{Outcome measures}

The mean frequencies of MMDs and migraine responder rates (MRRs) were calculated from eDiary data over 4-week intervals (Weeks 1-4; 5-8; 9-12; 13-16; $17-20 ; 21-24)$. A migraine responder was classified as a patient who achieved the specified reduction $(<25 \%$, $25-<50 \%, 50-<75 \%$, and $\geq 75 \%)$ in MMDs. The baseline frequency of migraine days was then compared to the migraine frequency in the 4-week intervals.

Patients also completed patient-reported outcome measures during the study, including the PGIC, which were evaluated at monthly intervals. The PGIC asked a single question concerning the patient's impression of the overall change in their disease status since the start 
of the study, which was rated on a 7-category scale ranging from "very much improved" to "very much worse."

For this post hoc analysis, patients were grouped by Month 1 response category (i.e., $<25 \%, 25-<50 \%, 50-<$ $75 \%$, and $\geq 75 \% \mathrm{MRR}$ ), and the number of subsequent study months with $\geq 50 \%$ and $\geq 75 \%$ MRR was calculated for each subgroup. The predictive ability of Month 1 PGIC ratings (very much improved, much improved, minimally improved, and no change/worse) were evaluated to determine which patients returned a PGIC response of much or very much improved at Month 6 . Patients were also grouped by Month 1 PGIC response to assess the frequency of PGIC responses of much or very much improved during the subsequent study months.

\section{Statistical analyses}

The full analysis set comprised all patients who received study medication. For PGIC calculations, only patients with responses at all specified timepoints were included. All analyses were conducted with SAS software (SAS Institute, Inc., Cary, NC, USA) version 9.2 or higher.

Monthly results (MMDs and MRRs) were calculated using the 4-week eDiary data intervals. If the eDiary was completed for at least 21 but less than 28 days, the observed frequency was normalized to the full 28day period. For patients with eDiary data for less than 21 of 28 days, the results were proportionally weighted based on the observed data plus data from the previous 4-week interval.

By comparing the baseline frequency of migraine days to the migraine frequency in each 4-weekly interval, it was possible to calculate changes; by determining a percent change from baseline, the migraine responder status $(<25 \%, 25-<50 \%, 50-<75 \%$, and $\geq$ $75 \%$ ) could be obtained. Initial responses (MRR and PGIC) were based on Month 1 data; response during the subsequent study months was based upon data reported during Months 2-6 (i.e., 5-8, 9-12, 13-16, $17-20$, and 21-24 weeks).

\section{Results}

A total of 1072 adults with chronic migraine participated in PROMISE-2 (mean age, 40.5 years; $88.2 \%$ female; 91.0\% white) [16]. This included 431 (40.2\%) with medication-overuse headache. Of the 1072 patients treated, 356 (33.2\%) received eptinezumab $100 \mathrm{mg}, 350$ (32.6\%) received eptinezumab $300 \mathrm{mg}$, and 366 (34.1\%) received placebo.

\section{Monthly migraine responder rates}

Over 6 study months, more patients treated with eptinezumab $100 \mathrm{mg}$ or $300 \mathrm{mg}$ were $\geq 50 \%$ or $\geq 75 \%$ migraine responders compared with patients receiving placebo (Fig. 1). In eptinezumab-treated patients, $\geq 50 \%$ migraine responder rates were generally consistent from Month 1 (100 mg, 194/356, 54.5\%; $300 \mathrm{mg}$, $212 / 350,60.6 \%)$ to Month 6 (100 mg, 212/356, 59.6\%; $300 \mathrm{mg}, 222 / 350,63.4 \%$ ), with placebo patients showing a slight increase over time (Month 1, 132/366, $36.1 \%$; Month $6,180 / 366,49.2 \%)$. The $\geq 75 \%$ migraine responder rates increased for all treatment arms from Month 1 (100 mg, 110/356, 30.9\%; $300 \mathrm{mg}, 129 / 350$, $36.9 \%$; placebo, $57 / 366,15.6 \%)$ to Month $6(100 \mathrm{mg}$, $143 / 356,40.2 \%$; $300 \mathrm{mg}, 164 / 350$, 46.9\%; placebo, $113 /$ $366,30.9 \%)$.

\section{Migraine response during month 1}

In the eptinezumab $100 \mathrm{mg}, 300 \mathrm{mg}$, and placebo groups, respectively, 194/356 (54.5\%), 212/350 $(60.6 \%)$, and $132 / 366(36.1 \%)$ patients were $\geq 50 \% \mathrm{mi}-$ graine responders (i.e., had $\mathrm{a} \geq 50 \%$ reduction in MMDs) during Month 1 (Table 1). Although rates of $50-<75 \%$ migraine response were similar across treatment groups (eptinezumab $100 \mathrm{mg}, 84 / 356$ [23.6\%]; $300 \mathrm{mg}, 83 / 350$ [23.7\%]; placebo, 75/366 [20.5\%]), more eptinezumab-treated than placebo-treated patients were $\geq 75 \%$ migraine responders $(100 \mathrm{mg}, 110 /$ 356 [30.9\%]; $300 \mathrm{mg}, 129 / 350$ [36.9\%]; placebo, 57/366 [15.6\%]). Indeed, in the eptinezumab groups, most patients who were $\geq 50 \%$ migraine responders were also $\geq 75 \%$ migraine responders (100 mg, $110 / 194$ [56.7\%]; $300 \mathrm{mg}, 129 / 212$ [60.8\%]; placebo, 57/132 [43.2\%]). Conversely, more placebo-treated than eptinezumabtreated patients were $25-<50 \%$ migraine responders (100 mg, 59/356 [16.6\%]; $300 \mathrm{mg}, 58 / 350$ [16.6\%]; placebo, $81 / 366[22.1 \%]$ ) or $<25 \%$ migraine responders (103/356 [28.9\%]; 80/350 [22.9\%]; $153 / 366$ [41.8\%], respectively).

During Month 1, $\geq 75 \%$ migraine responders demonstrated a mean (standard deviation) reduction in MMDs of approximately $90 \%$ (eptinezumab $100 \mathrm{mg},-89.0 \%$ [8.34]; $300 \mathrm{mg},-90.8 \%$ [8.55]; placebo, $-87.3 \%$ [8.58]). MMD reductions in the $50-<75 \%, 25-<50 \%$, and $<25 \%$ migraine responder subgroups were approximately $62 \%$, $36 \%$, and $4 \%$, respectively.

Baseline characteristics according to Month 1 migraine responses are shown in Table 2. No notable differences in demographic or clinical variables were observed between patients in each quartile, suggesting that none of these baseline factors influenced migraine response during Month 1.

\section{MRR during subsequent study months}

Response trajectories based on Month 1 response are shown in Fig. 2. Consistency of Month 1 data during 


\section{A. Monthly $\geq 50 \%$ migraine responder rates}

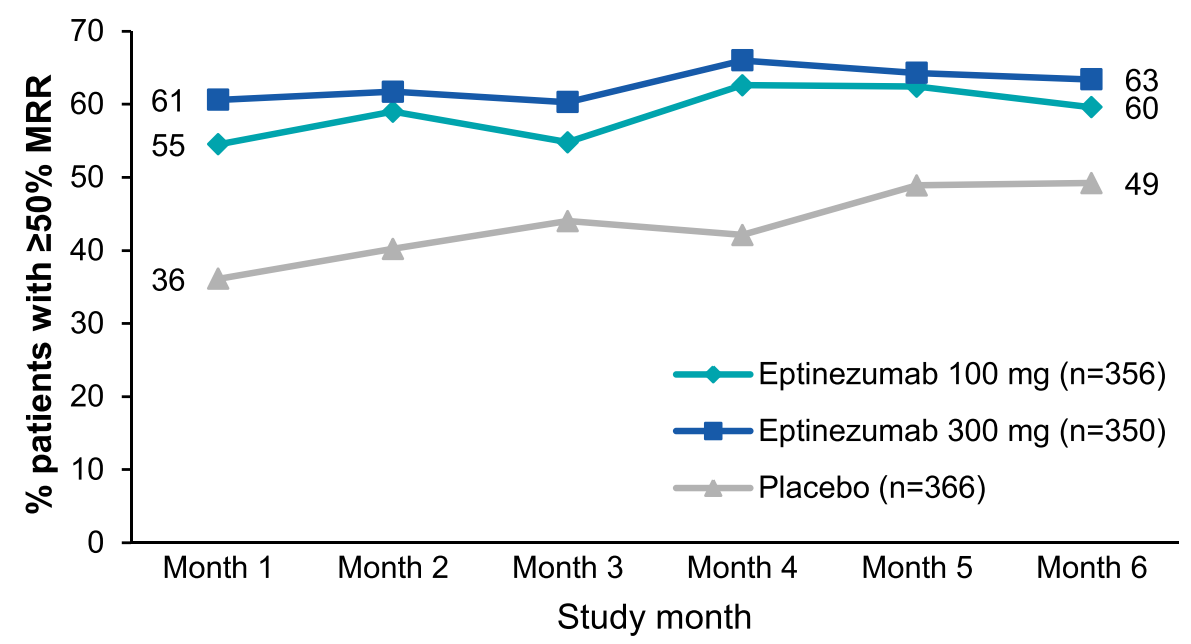

\section{B. Monthly $\geq 75 \%$ migraine responder rates}

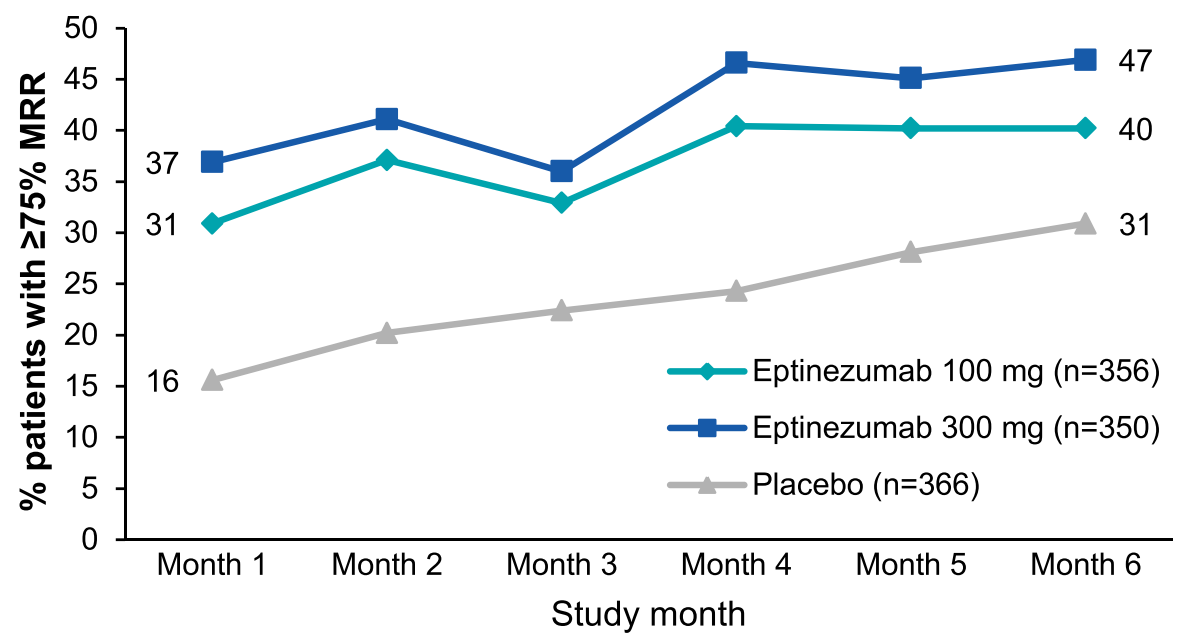

Fig. 1 Monthly migraine responder ${ }^{\mathrm{a}}$ rates: $(\mathbf{A}) \geq 50 \%$ and $(\mathbf{B}) \geq 75 \%$. ${ }^{\mathrm{a}}$ Migraine response was defined as a reduction in monthly migraine days. MRR, migraine responder rate

the subsequent study months, according to response group, is shown in Figs. 3 and 4 .

Most patients who achieved $\geq 75 \%$ migraine response during Month 1 subsequently experienced $\geq 75 \%$ migraine response over each study month (Month 2, 215/296, 72.6\%; Month 3, 192/296, 64.9\%; Month 4, 217/296, 73.3\%; Month 5, 212/296, 71.6\%; Month 6, 205/296, 69.3\%) (Fig. 2 A). Among patients who achieved $\geq 75 \%$ migraine response in Month 1 , more than one-third (127/296 [42.9\%]) continued to attain $\geq 75 \%$ migraine response for all 5 subsequent study months (eptinezumab $100 \mathrm{mg}, 46 / 110$ [41.8\%]; $300 \mathrm{mg}, 60 / 129$ [46.5\%]; placebo, 21/57 [36.8]\%) (Fig. 3 A). More than two-thirds of Month $1 \geq 75 \%$ responders achieved $\geq 75 \%$ migraine response for $\geq 3$ subsequent months (80/110 [72.7\%]; 98/129 [76.0\%]; and 38/57 [66.7\%], respectively) (Fig. 3 A). In addition, among the patients who achieved $\geq 75 \% \mathrm{mi}-$ graine response in Month 1, nearly all (293/296 [99.0\%]) had at least 1 month of $\geq 50 \%$ migraine response during the subsequent 5 months, and more than two-thirds $(210 / 296$ [70.9\%]) had a $\geq 50 \% \mathrm{mi}$ graine response for all 5 subsequent study months (100 mg, 76/110 [69.1\%]; $300 \mathrm{mg}, 96 / 129$ [74.4\%]; placebo, 38/ 57 [66.7\%]) (Fig. 4 A).

Most patients who achieved $50-<75 \%$ migraine response during Month 1 subsequently experienced $\geq 50 \%$ migraine response over each study month (Month 2, 176/242, 72.7\%; Month 3, 161/242, 66.5\%; Month 4, 172/242, 71.1\%; Month 5, 170/242, 70.2\%; Month 6, 
Table 1 MRR and PGIC at Month 1

\begin{tabular}{|c|c|c|c|}
\hline & Eptinezumab $100 \mathrm{mg}$ & Eptinezumab $300 \mathrm{mg}$ & Placebo \\
\hline \multicolumn{4}{|l|}{ MRR } \\
\hline$n$ & 356 & 350 & 366 \\
\hline$\geq 75 \%$ & $110(30.9)$ & $129(36.9)$ & $57(15.6)$ \\
\hline $50-<75 \%$ & 84 (23.6) & $83(23.7)$ & $75(20.5)$ \\
\hline $25-<50 \%$ & 59 (16.6) & $58(16.6)$ & $81(22.1)$ \\
\hline$<25 \%$ & $103(28.9)$ & $80(22.9)$ & $153(41.8)$ \\
\hline \multicolumn{4}{|l|}{ PGIC } \\
\hline$n^{*}$ & 328 & 329 & 330 \\
\hline Very Much Improved & $41(12.5)$ & $81(24.6)$ & $27(8.2)$ \\
\hline Much Improved & $110(33.5)$ & $112(34.0)$ & $80(24.2)$ \\
\hline Minimally Improved & $103(31.4)$ & $76(23.1)$ & $82(24.8)$ \\
\hline No Improvement ${ }^{\dagger}$ & $74(22.6)$ & $60(18.2)$ & $141(42.7)$ \\
\hline
\end{tabular}

*Only patients with data at Month 1 and Month 6 are included

†No improvement includes "no change," "minimally worse," "much worse," and "very much worse"

MRR, migraine response rate; PGIC, Patient Global Impression of Change

166/242, 68.6\%) (Fig. 2 B). During Months 2 and 3, the proportion of $\geq 75 \%$ and $50-75 \%$ response was similar; during Months 4 through 6, more patients were $\geq 75 \%$ responders than $\geq 50 \%$ responders. Among patients who achieved $50-<75 \%$ migraine response in Month 1 , more than one-third $(104 / 242[43.0 \%])$ achieved $\geq 50 \% \mathrm{mi}$ graine response for all 5 subsequent study months (100 mg, 36/84 [42.9\%]; $300 \mathrm{mg}, 31 / 83$ [37.3\%]; placebo, 37/ 75 [49.3\%]) and more than two-thirds achieved $\geq 50 \%$ migraine response for $\geq 3$ months (63/84 [75.0\%]; 58/83 [69.9\%]; and 53/75 [70.7\%], respectively) (Fig. 4 B).

Three times as many eptinezumab-treated patients who were $25-<50 \%$ migraine responders in Month 1 achieved $\geq 50 \%$ migraine response for all 5 subsequent study months compared with placebo (eptinezumab 100 mg, 14/59 [23.7\%]; $300 \mathrm{mg}, 15 / 58$ [25.9\%]; placebo, 5/81 [6.2\%]) (Fig. 4 C). Overall, about half of patients in this Month 1 response group (95/198 [48.0\%]) still achieved $\geq 3$ study months with $\geq 50 \%$ migraine response $(100 \mathrm{mg}$, 31/59 [52.5\%]; $300 \mathrm{mg}, 30 / 58$ [51.7\%]; placebo, 34/81 [42.0\%]).

Among patients with $<25 \%$ migraine response during Month 1, 8/103 (7.8\%), 6/80 (7.5\%), and 6/153 (3.9\%) of patients in the eptinezumab $100 \mathrm{mg}, 300 \mathrm{mg}$, and placebo groups, respectively, achieved $\geq 50 \% \mathrm{mi}-$ graine response for all 5 subsequent study months, while 52/103 (50.5\%), 33/80 (41.3\%), and 61/153 (39.9\%), respectively, achieved $\geq 1$ subsequent study month with $\geq 50 \%$ migraine response (Fig. 4 D). More eptinezumab-treated than placebo-treated patients with $<25 \%$ migraine response during Month 1 achieved $\geq 3$ subsequent months of $\geq 50 \%$ migraine response (eptinezumab $100 \mathrm{mg}, 24 / 103$ [23.3\%]; $300 \mathrm{mg}$, 20/80 [25.0\%]; placebo, 21/153 [13.7\%]).
PGIC ratings at month 1 and PGIC response during the subsequent study months

Of patients with PGIC data available at both Month 1 and Month 6, 151/328 (46.0\%) of those who received eptinezumab $100 \mathrm{mg}$ and 193/329 (58.7\%) who received eptinezumab $300 \mathrm{mg}$ indicated that their condition was very much or much improved at Month 1 (Table 1). In contrast, $107 / 330(32.4 \%)$ of those who received placebo were very much or much improved at Month 1. Of the remaining placebo-treated patients, $82 / 330(24.8 \%)$ were minimally improved, and $141 / 330(42.7 \%)$ indicated no improvement at Month 1.

PGIC ratings of very much improved or much improved at Month 1 were predictive of PGIC ratings at Month 6. More than $80 \%$ of the subgroup of patients who were very much improved at Month 1 were very much improved or much improved at Month 6 (82.9\%, 86.4\%, and $81.5 \%$ for eptinezumab $100 \mathrm{mg}$, eptinezumab $300 \mathrm{mg}$, and placebo, respectively). For the subgroup of patients who were much improved at Month 1, rates of very much improved or much improved at Month 6 were 71.8\%, 75.9\%, and 66.3\%, respectively.

Of patients with PGIC data at all timepoints, more than $90 \%$ of the very much improved and much improved subgroups during Month 1 had at least 1 subsequent month of the same response level. More than two-thirds of those in the very much improved subgroup were very much improved or much improved for all 5 subsequent months, and approximately half of those in the much improved subgroup were very much improved or much improved for all 5 subsequent study months (Fig. 5). 


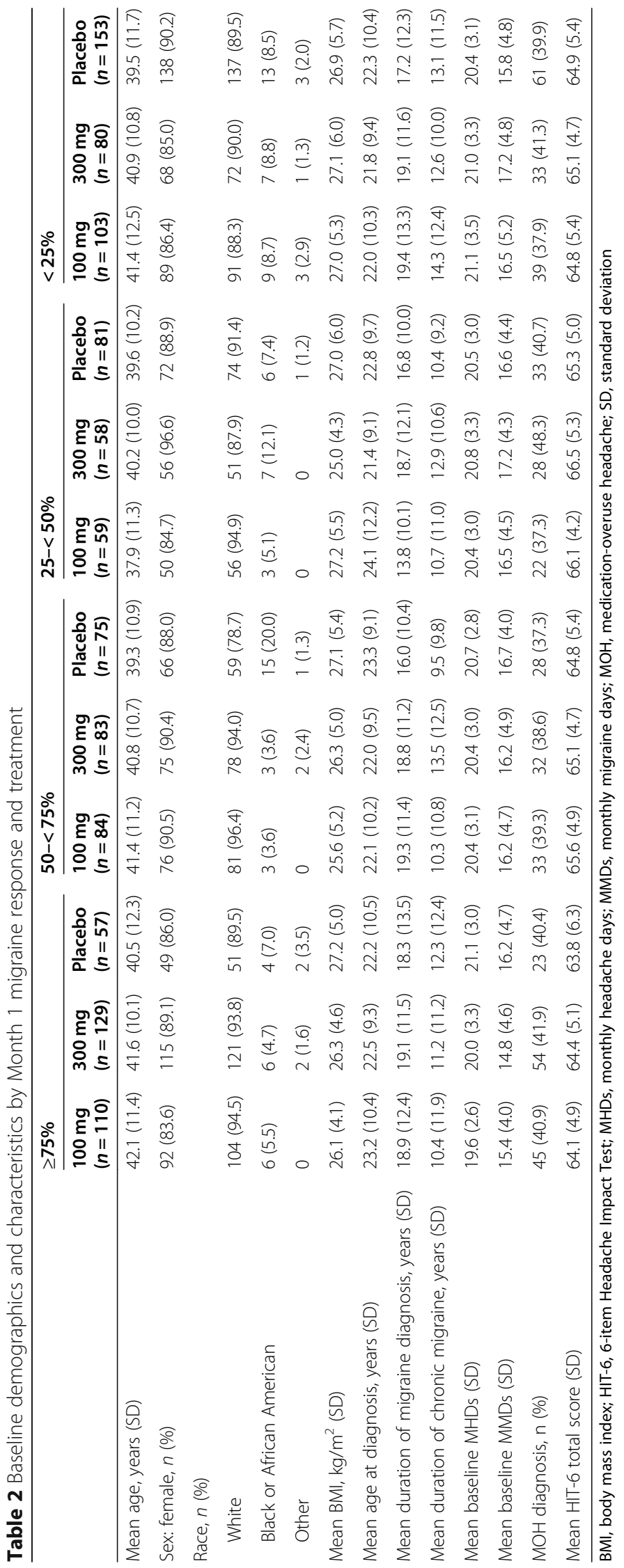




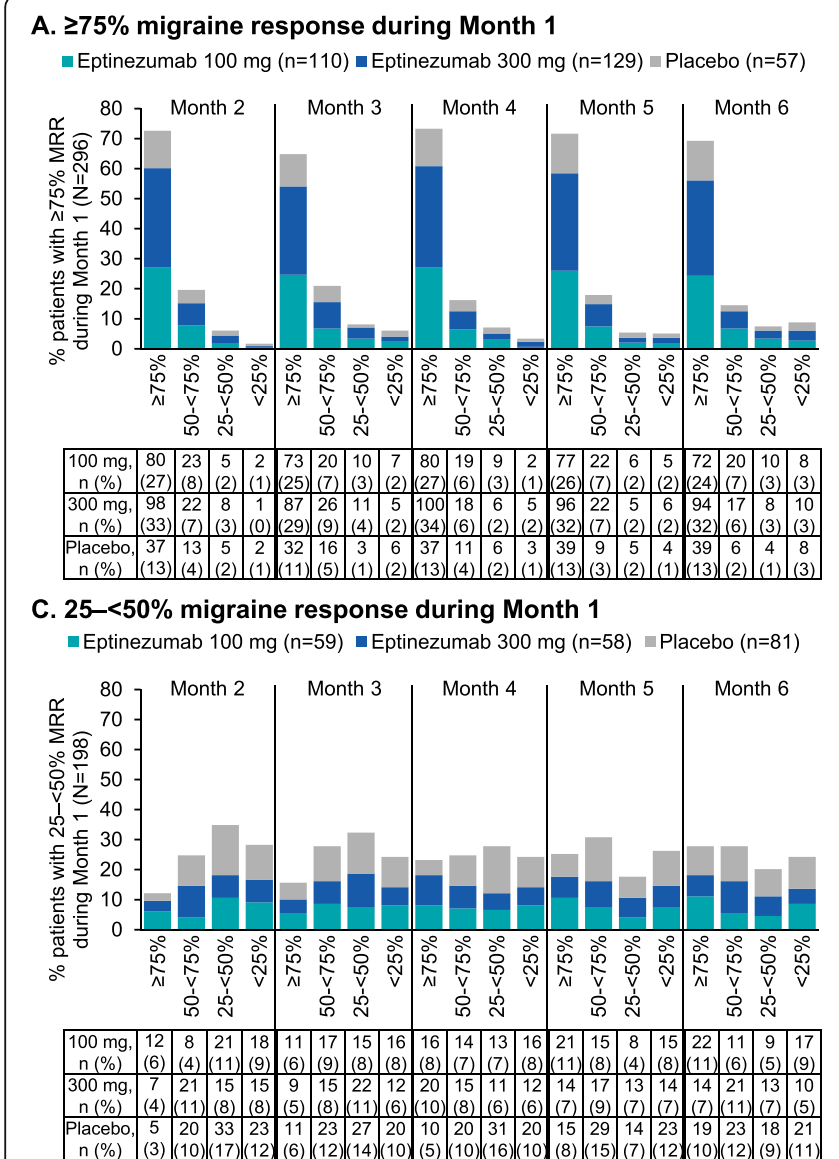

B. $50-<75 \%$ migraine response during Month 1

=Eptinezumab $100 \mathrm{mg}(\mathrm{n}=84)$ Eptinezumab $300 \mathrm{mg}(\mathrm{n}=83)$-Placebo $(\mathrm{n}=75)$

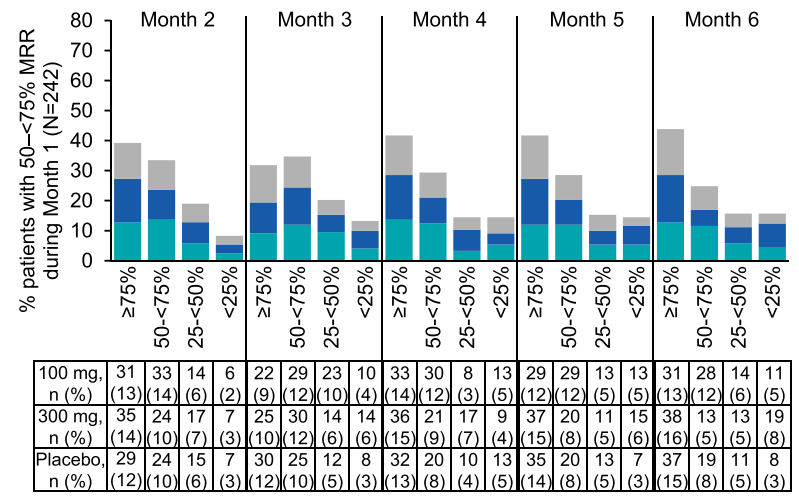

D. $<25 \%$ migraine response during Month 1

- Eptinezumab $100 \mathrm{mg}(\mathrm{n}=103)$ - Eptinezumab $300 \mathrm{mg}(\mathrm{n}=80)$ Placebo $(\mathrm{n}=153)$

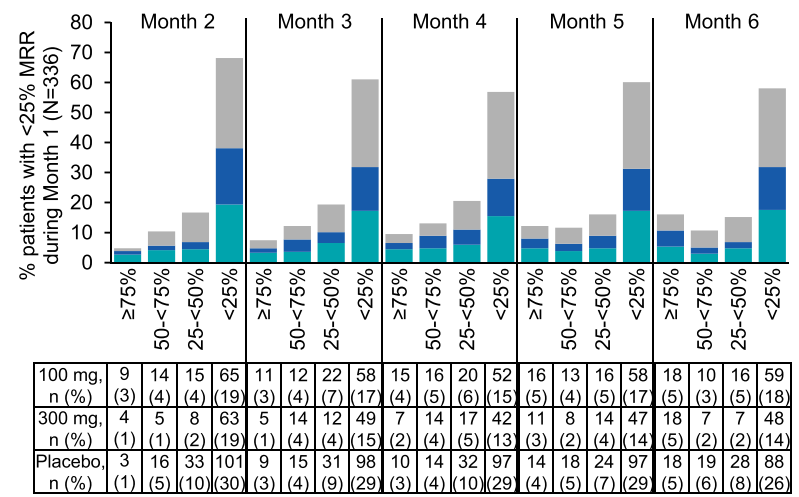

Fig. 2 Monthly response according to Month 1 migraine response ${ }^{a}$ category: (A) $\geq 75 \%$, (B) $50-<75 \%$, (C) $25-<50 \%$, (D) $<25 \%$. ${ }^{a}$ Migraine response was defined as a percentage reduction in monthly migraine days. MRR, migraine responder rate

\section{Discussion}

The ability to assess early-stage responses to a preventive migraine treatment and predict the subsequent response trajectory would better equip clinicians to manage patient expectations and improve therapeutic decision-making. In this post hoc analysis of the PROMISE-2 study in patients with chronic migraine, we evaluated the consistency and predictive ability of migraine response during Month 1 on later outcomes. Our data confirmed that more eptinezumab-treated than placebo-treated patients were $\geq 50 \%$ and $\geq 75 \%$ migraine responders during the entire study period, including Month 1, and revealed that an early response was generally predictive of a sustained response, with many patients experiencing consistent benefits during the subsequent months.

The aim of the analysis was to highlight the consistency and general predictive ability of eptinezumab preventive efficacy, which biologically and clinically has shown rapid onset of action [22, 23]; this, in turn, is expected to allow earlier clinical decisionmaking regarding future patient care. Eptinezumab treatment resulted in greater rates of migraine and PGIC response than placebo, and our data indicate that Month 1 response to treatment was predictive of response throughout the study, with most eptinezumab-treated patients who achieved Month 1 migraine response continuing to respond at the same level or higher for at least half of the 24-week treatment period. Although some other new migraine preventives have demonstrated an early onset of effect, this is, to our knowledge, the first analysis to describe the response trajectory based on the first month of treatment.

The American Headache Society recommends that clinicians help patients establish realistic expectations regarding the anticipated benefits of prescribed preventives, including effects on migraine and headache frequency, attack duration, attack severity, migrainerelated disability, psychological distress, acute treatment response, functioning in important areas of life, and/or health-related quality of life [3]. Identification of early non-response is also an opportunity for clinicians to advance patient management in a timely 


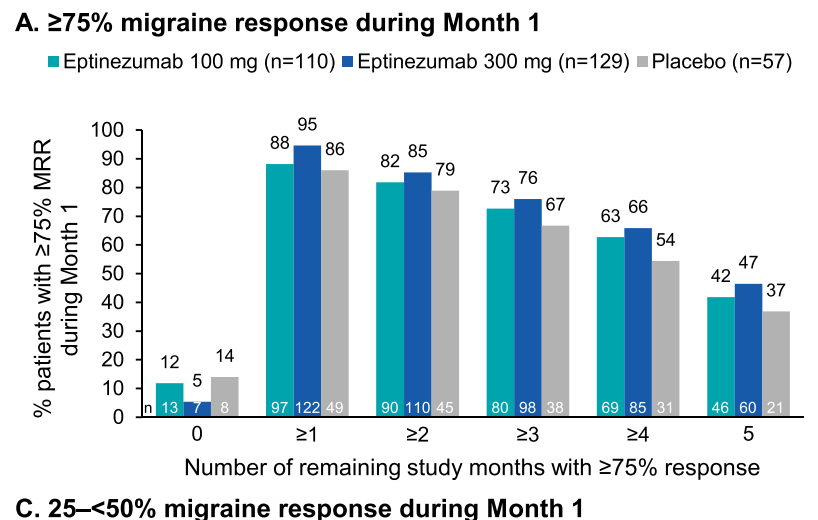

Eptinezumab $100 \mathrm{mg}(\mathrm{n}=59)$ - Eptinezumab $300 \mathrm{mg}(\mathrm{n}=58)$ Placebo $(\mathrm{n}=81)$

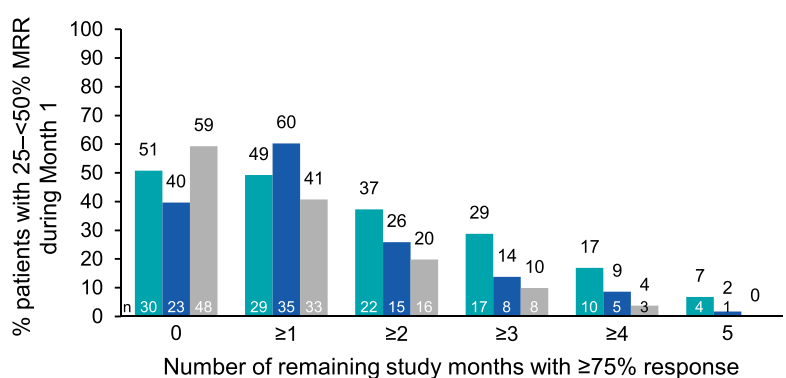

B. $50-<75 \%$ migraine response during Month 1

-Eptinezumab $100 \mathrm{mg}(\mathrm{n}=84)$-Eptinezumab $300 \mathrm{mg}(\mathrm{n}=83)$-Placebo $(\mathrm{n}=75)$

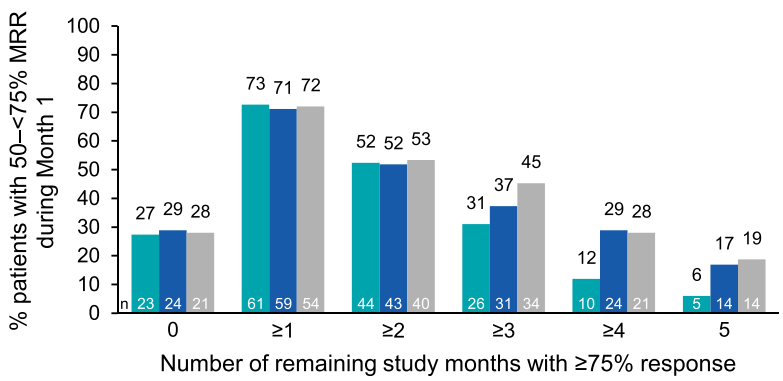

D. $<25 \%$ migraine response during Month 1

- Eptinezumab $100 \mathrm{mg}(\mathrm{n}=103)$ Eptinezumab $300 \mathrm{mg}(\mathrm{n}=80) \backsim$ Placebo $(n=153)$

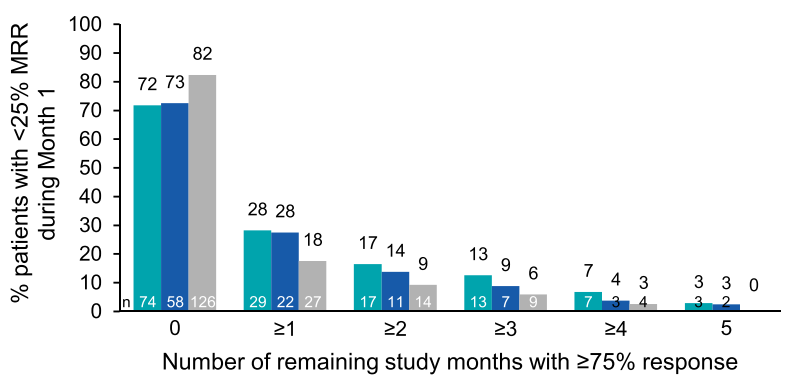

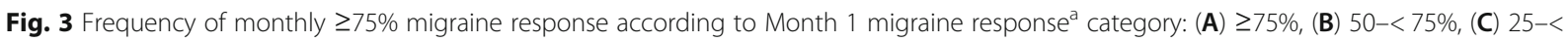
50\%, (D) < 25\%. 'Migraine response was defined as a reduction in monthly migraine days. MRR, migraine responder rate

manner. The results of the current analysis suggest that patients initiating eptinezumab may be able to recognize their response trajectories (with respect to migraine frequency and PGIC) earlier than patients beginning oral preventives ( 1 month vs $2-12$ months, respectively) [3]. This early onset and consistency of response is not unexpected, given the pharmacokinetics and mechanism of action of eptinezumab. Intravenous administration of eptinezumab ensures 100\% bioavailability, with maximum plasma concentration by the end of the infusion (i.e., $\sim 30 \mathrm{~min}$ ) [24]. In addition, eptinezumab has a highly potent and selective binding profile for the CGRP ligand [22] and a terminal elimination half-life of $\sim 27$ days [19]. These attributes are responsible for the early onset of efficacy (preventive efficacy on Day 1 after dosing [25] and symptomatic efficacy after $2 \mathrm{~h}$ when administered during a migraine attack [26]) previously reported for eptinezumab, the sustained effectiveness observed over 12 weeks post-dose $[16,27,28]$, and the persistent or increased effects reported with additional dosing [29].

The high rates of response to eptinezumab during Month 1 and the maintenance and consistency of the subsequent response profile could potentially help to provide guidance and/or a framework for management decisions for patients with chronic migraine. For clinicians, these decisions include whether to continue with preventive treatment, the necessity for interim prescription of acute medication, and recommendations for lifestyle modifications and bio-behavioral training. For patients, prediction of response may simplify future planning for their professional and social lives and provide more realistic treatment expectations according to the individuality of migraine phenotypes and the burden of disease. These results will likely also inspire hope and optimism in patients, which may have the effect of improving psychological symptoms and reducing migraine-related distress, although formal effects on health-related quality of life remain to be confirmed. To fully achieve treatment response prediction, additional studies are needed to explore relationships between the early onset of effect and other outcome measures, such as the Migraine Disability Assessment, 6-item Headache Impact Test, 36-item Short Form Health Survey, Migraine Interictal Burden Scale, Migraine-Specific Quality of Life Questionnaire, Patient-Identified Most Bothersome Symptom, acute headache medication use, and psychologic symptomatology. These additional studies should also account for the inherent month-to-month variability of migraine frequency as well as the administration of multiple doses, which were not explored in the current analysis. 


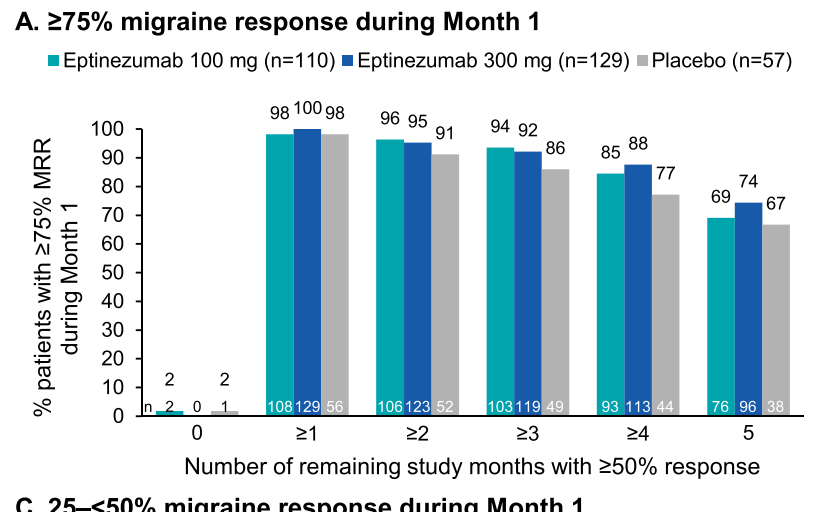

C. $25-<50 \%$ migraine response during Month 1

- Eptinezumab $100 \mathrm{mg}(\mathrm{n}=59)$ - Eptinezumab $300 \mathrm{mg}(\mathrm{n}=58)$-Placebo ( $\mathrm{n}=81)$

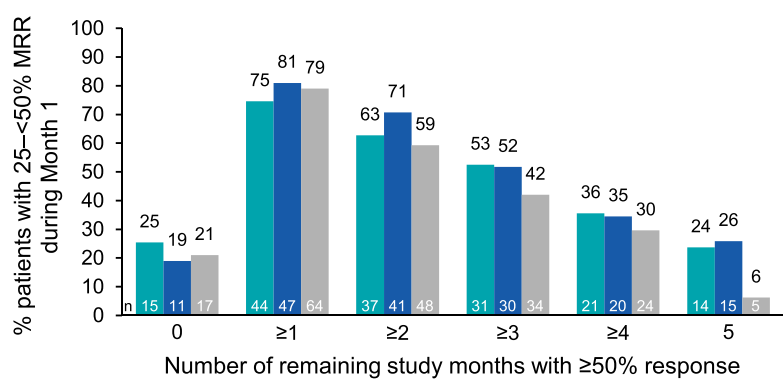

B. $50-<75 \%$ migraine response during Month 1

-Eptinezumab $100 \mathrm{mg}(\mathrm{n}=84)$-Eptinezumab $300 \mathrm{mg}(\mathrm{n}=83)$-Placebo $(\mathrm{n}=75)$

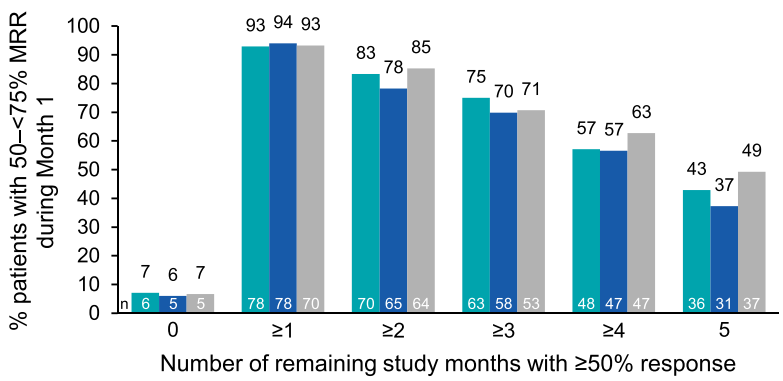

D. $<25 \%$ migraine response during Month 1

- Eptinezumab $100 \mathrm{mg}(\mathrm{n}=103)$ - Eptinezumab $300 \mathrm{mg}(\mathrm{n}=80)$ - Placebo $(n=153)$

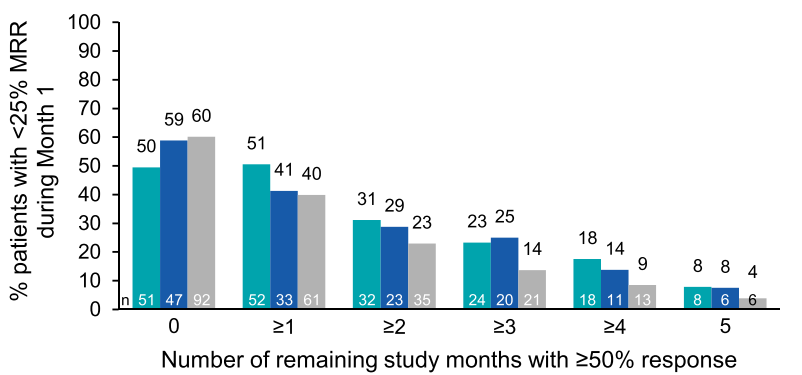

Fig. 4 Frequency of monthly $\geq 50 \%$ migraine response according to Month 1 migraine response ${ }^{a}$ : (A) $\geq 75 \%$, (B) $50-<75 \%$, (C) $25-<50 \%$, (D) $<25 \%$. ${ }^{a}$ Migraine response was defined as a percentage reduction in monthly migraine days. MRR, migraine responder rate

It is clear that early response with eptinezumab has predictive value for subsequent response. However, our data also indicated that some patients who were early non-responders at Month 1 could still become responders in subsequent time periods. Approximately $50 \%$ and $25 \%$ of eptinezumab-treated patients with $25-<50 \%$ and $<25 \%$ migraine response during Month 1 , respectively, achieved $\geq 50 \%$ migraine response for at least half of the 6-month treatment period (vs $42 \%$ and $14 \%$ with placebo, respectively). This indicates the importance of not halting or switching eptinezumab treatment too early and underscores the need for appropriate interim migraine management, as well as management of patient education and expectation.

Limitations of this study include the post hoc design of the analyses, which necessitates additional studies to confirm these findings and to identify the predictive value of early response with respect to other outcomes. Notably, due to the heterogeneous nature of migraine [30], the MMD measure has inherent month-to-month variability, and we consider that this may have reduced the Month 1 predictive ability. That is, for patients who were naturally experiencing relative improvements during the course of their chronic migraine, smaller therapeutic benefit (impact of eptinezumab on MMDs) would be observed, whereas for patients experiencing relative worsening of migraine at the time of the study, the beneficial impact of eptinezumab may have been overemphasized. As in many previous clinical trials in migraine patients, there was a high placebo response rate in this phase 3 study; however, effectiveness in the placebo group remained consistently lower than that of eptinezumab in terms of response rates and PGIC improvement. Finally, based on the demographic characteristics that were reported in the full study population (i.e., predominantly white and nonHispanic) [16], the results may not be generalizable to all patients with chronic migraine, with further research warranted in more racially and ethnically diverse patient groups.

\section{Conclusion}

In this post hoc analysis of data from PROMISE-2, more eptinezumab-treated than placebo-treated patients were $\geq 75 \%$ MRR responders during Month 1 . The majority of eptinezumab-treated patients who achieved a migraine response during Month 1 went on to achieve the same or a higher level of response for at least half of the entire 24-week treatment period. However, the potential for later response in early eptinezumab non-responders was also observed. These findings could help clinicians and patients set 


\section{A. PGIC rating of "very much improved" at Month 1}

-Eptinezumab $100 \mathrm{mg}(\mathrm{n}=40)$-Eptinezumab $300 \mathrm{mg}(\mathrm{n}=79)$-Placebo $(n=25)$

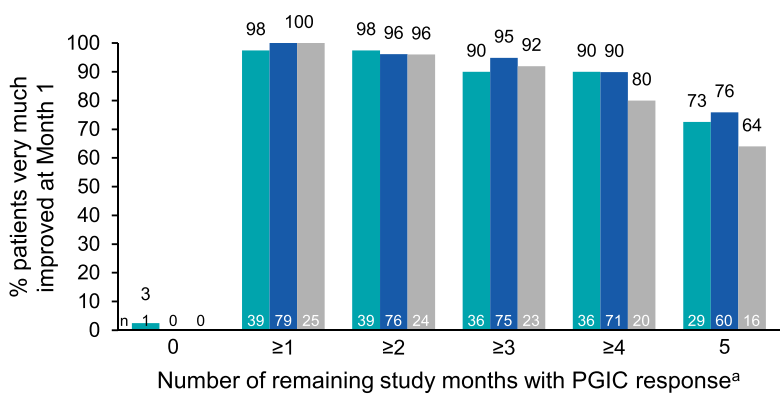

C. PGIC rating of "minimally improved" at Month 1

-Eptinezumab $100 \mathrm{mg}(\mathrm{n}=101)$-Eptinezumab $300 \mathrm{mg}(\mathrm{n}=74) \backsim$ Placebo $(n=81)$

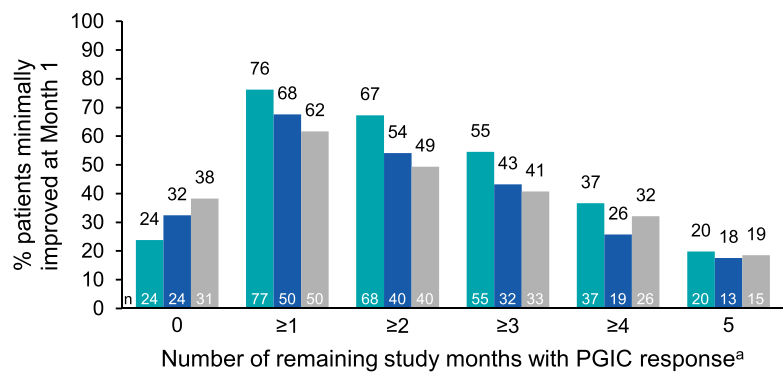

\section{B. PGIC rating of "much improved" at Month 1}

- Eptinezumab $100 \mathrm{mg}(\mathrm{n}=110)$-Eptinezumab $300 \mathrm{mg}(\mathrm{n}=110) \backsim$ Placebo $(\mathrm{n}=76)$

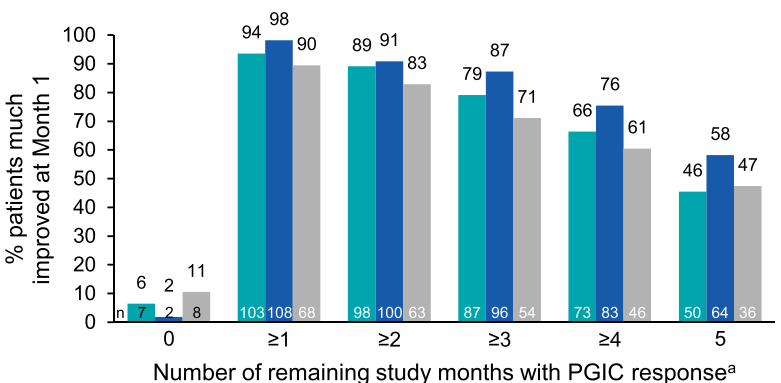

\section{PGIC rating of "not improved" at Month $1^{\mathrm{b}}$}

- Eptinezumab $100 \mathrm{mg}(\mathrm{n}=69)$ - Eptinezumab $300 \mathrm{mg}(\mathrm{n}=58) \backsim$ Placebo $(n=137)$

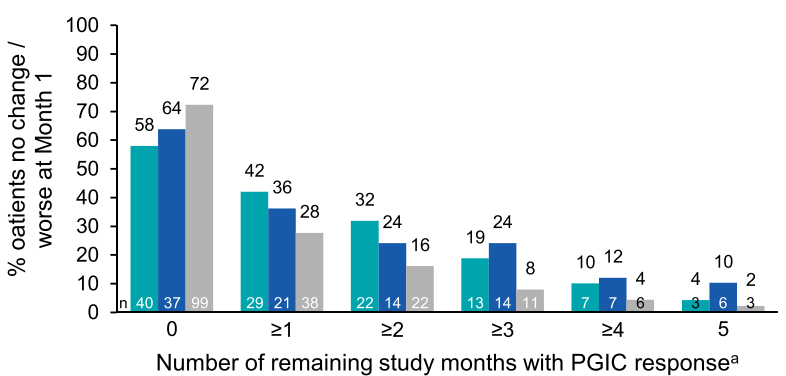

Fig. 5 Frequency of PGIC response a according to PGIC rating at Month 1: (A) very much improved, (B) much improved, (C) minimally improved, (D) no improvement. "PGIC response defined as rating of "much improved" or "very much improved." bIncludes patients reporting "no change," "minimally worse," "much worse," and "very much worse." Analysis conducted in patients with PGIC data at all time points. PGIC, Patient Global Impression of Change

realistic treatment expectations and accelerate appropriate management decisions.

\section{Abbreviations}

CGRP: Calcitonin gene-related peptide; eDiary: Electronic diary; ICHD3ß: International Classification of Headache Disorders, 3rd edition, beta version; MMDs: Monthly migraine days; MRR: Migraine responder rate; PGIC: Patient Global Impression of Change

\section{Acknowledgments}

The authors thank Nicole Coolbaugh, CMPP, and Sally-Anne Mitchell, PhD, of The Medicine Group, LLC (New Hope, PA, USA) for providing medical writing support, which was funded by H. Lundbeck A/S (Copenhagen, Denmark) in accordance with Good Publication Practice guidelines.

\section{Authors' contributions}

DCB, PKW, LCIV, TB, and RC contributed to the conception and design of the study or data acquisition. JH performed the statistical analyses, and all authors contributed to interpretation of the data. All authors reviewed and provided critical revision of all manuscript drafts for important intellectual content, as well as read and approved the final manuscript for submission.

\section{Funding}

Sponsored and funded by $\mathrm{H}$. Lundbeck A/S. The sponsor participated in the design and conduct of the study; data collection, management, analysis, and interpretation; and preparation, review, and approval of the manuscript. All statistical analyses were performed by a contracted research organization and were directed or designed by Pacific Northwest Statistical Consulting under contractual agreement with $\mathrm{H}$. Lundbeck $\mathrm{A} / \mathrm{S}$. All authors and $\mathrm{H}$. Lundbeck A/S prepared, reviewed, and approved the manuscript and made the decision to submit the manuscript for publication. Editorial support for the development of this manuscript was funded by H. Lundbeck A/S.

\section{Availability of data and materials}

In accordance with EFPIA's and PhRMA's "Principles for Responsible Clinical Trial Data Sharing" guidelines, Lundbeck is committed to responsible sharing of clinical trial data in a manner that is consistent with safeguarding the privacy of patients, respecting the integrity of national regulatory systems, and protecting the intellectual property of the sponsor. The protection of intellectual property ensures continued research and innovation in the pharmaceutical industry. Deidentified data is available to those whose request has been reviewed and approved through an application submitted to https://www.lundbeck.com/global/our-science/clinical-data-sharing.

\section{Declarations}

Ethics approval and consent to participate

Approval for PROMISE-2 was provided by the independent ethics committee or institutional review board of the study sites. PROMISE-2 was conducted in accordance with Good Clinical Practice guidelines, the principles of the Declaration of Helsinki, and all applicable regulatory requirements. Patients provided written informed consent prior to initiation of any study procedures.

\section{Consent for publication}

Not applicable.

\section{Competing interests}

DCB: Received grant support from Amgen, the National Headache Foundation and the FDA; received consulting support from Allergan/Abbvie, Amgen, Lilly, Lundbeck, and Teva; and serves on the editorial board of Current Pain and Headache Reports.

PKW: Receives consulting fees from Abbvie, Aeon, Amgen, Axsome, Biohaven, GlaxoSmithKline, Impel, Lilly, Lundbeck, Nesos, Satsuma, Theranica, and Teva; serves on speaker bureaus for Abbvie, Amgen, Biohaven, Lilly, Lundbeck, Novartis, Teva; and receives research support from AbbVie, Biohaven, Lilly, Satsuma, and Zosano. 
LCIV: Receives personal compensation for serving as a consultant for Alder/ Lundbeck, Allergan/AbbVie, Biohaven, Satsuma, and Teva and for serving as an Expert Witness for Vaccine Injury Compensation Program. He is on the advisory panel of Ctrl M Health (stock). In addition, he is a non-compensated Associate Editor with Headache and has a non-compensated relationship as a Board Member at Large with Alliance for Headache Disorders Advocacy. $\mathrm{JH}$ : Employee of Pacific Northwest Statistical Consulting, Inc., a contracted service provider of biostatistical resources for $\mathrm{H}$. Lundbeck A/S.

RC: Employee of Lundbeck or one of its subsidiary companies at the time of study and manuscript development.

TB: Employee of $\mathrm{H}$. Lundbeck A/S or one of its subsidiary companies and has equity in $\mathrm{H}$. Lundbeck $\mathrm{A} / \mathrm{S}$.

\section{Author details}

${ }^{1}$ Albert Einstein College of Medicine, Bronx, NY, USA. ${ }^{2}$ Vector Psychometric Group, LLC, Chapel Hill, NC, USA. ${ }^{3}$ Palm Beach Headache Center, West Palm Beach, FL, USA. ${ }^{4}$ Department of Neurology and Ophthalmology, Michigan State University College of Human Medicine, East Lansing, MI, USA. ${ }^{5}$ Pacific Northwest Statistical Consulting, Inc., Woodinville, WA, USA. ' $L$ Lundbeck LLC, IL, Deerfield, USA. ${ }^{7} \mathrm{H}$. Lundbeck A/S, Copenhagen, Denmark.

\section{Received: 1 October 2021 Accepted: 6 January 2022} Published online: 21 February 2022

\section{References}

1. Headache Classification Committee of the International Headache Society (IHS) (2018) The international classification of headache disorders, 3rd edition. Cephalalgia 38(1):1-211. https://doi.org/10.1177/0333102417738202

2. Adams AM, Serrano D, Buse DC, Reed ML, Marske V, Fanning KM, Lipton RB (2015) The impact of chronic migraine: the chronic migraine epidemiology and outcomes (CaMEO) study methods and baseline results. Cephalalgia. 35(7):563-578. https://doi.org/10.1177/0333102414552532

3. Ailani J, Burch RC, Robbins MS (2021) The American headache society consensus statement: update on integrating new migraine treatments into clinical practice. Headache. 61(7):1021-1039. https:/doi.org/10.1111/head.14153

4. Aurora SK, Dodick DW, Diener HC, DeGryse RE, Turkel CC, Lipton RB et al (2014) OnabotulinumtoxinA for chronic migraine: efficacy, safety, and tolerability in patients who received all five treatment cycles in the PREEMPT clinical program. Acta Neurol Scand 129(1):61-70. https://doi.org/10.1111/ane.12171

5. Bigal ME, Dodick DW, Krymchantowski AV, VanderPluym JH, Tepper SJ, Aycardi E et al (2016) TEV-48125 for the preventive treatment of chronic migraine: efficacy at early time points. Neurology. 87(1):41-48. https://doi. org/10.1212/wnl.0000000000002801

6. Brandes J, Yeung PP, Aycardi E, Bigal M, Blankenbiller T, Grozinski-Wolff M et al (2018) Early onset of action with fremanezumab versus placebo for the preventive treatment of episodic migraine (P4.107). Neurology. 90(15 Supplement):P4.107

7. Camporeale A, Kudrow D, Sides R, Wang S, Van Dycke A, Selzler KJ et al (2018) A phase 3, long-term, open-label safety study of Galcanezumab in patients with migraine. BMC Neurol 18(1):188. https://doi.org/10.1186/s12 883-018-1193-2

8. Detke HC, Goadsby PJ, Wang S, Friedman DI, Selzler KJ, Aurora SK (2018) Galcanezumab in chronic migraine: the randomized, double-blind, placebocontrolled REGAIN study. Neurology. 91(24):e2211-e2221. https://doi.org/1 0.1212/wnl.0000000000006640

9. Detke HC, Millen BA, Zhang Q, Samaan K, Ailani J, Dodick DW, Aurora SK (2020) Rapid onset of effect of galcanezumab for the prevention of episodic migraine: analysis of the EVOLVE studies. Headache. 60(2):348-359. https:// doi.org/10.1111/head.13691

10. Dodick DW, Ashina M, Brandes JL, Kudrow D, Lanteri-Minet M, Osipova V , Palmer K, Picard H, Mikol DD, Lenz RA (2018) ARISE: a phase 3 randomized trial of erenumab for episodic migraine. Cephalalgia. 38(6):1026-1037. https://doi.org/10.1177/0333102418759786

11. Dodick DW, Silberstein SD, Lipton RB, DeGryse RE, Adams AM, Diener HC (2019) Early onset of effect of onabotulinumtoxinA for chronic migraine treatment: analysis of PREEMPT data. Cephalalgia. 39(8):945-956. https://doi. org/10.1177/0333102418825382

12. Dodick DW, Silberstein SD, Bigal ME, Yeung PP, Goadsby PJ, Blankenbiller T, Grozinski-Wolff M, Yang R, Ma Y, Aycardi E (2018) Effect of fremanezumab compared with placebo for prevention of episodic migraine: a randomized clinical trial. Jama. 319(19):1999-2008. https://doi.org/10.1001/jama.2018.4853
13. Forderreuther S, Zhang Q, Stauffer VL, Aurora SK, Lainez MJA (2018) Preventive effects of galcanezumab in adult patients with episodic or chronic migraine are persistent: data from the phase 3, randomized, double-blind, placebo-controlled EVOLVE-1, EVOLVE-2, and REGAIN studies. J Headache Pain. 19(1):121. https://doi.org/10.1186/s10194-018-0951-2

14. Goadsby PJ, Reuter U, Hallstrom Y, Broessner G, Bonner JH, Zhang F et al (2017) A controlled trial of erenumab for episodic migraine. N Engl J Med 377(22):2123-2132. https://doi.org/10.1056/NEJMoa1705848

15. Goadsby PJ, Dodick DW, Martinez JM, Ferguson MB, Oakes TM, Zhang Q, Skljarevski $V$, Aurora SK (2019) Onset of efficacy and duration of response of galcanezumab for the prevention of episodic migraine: a post-hoc analysis. J Neurol Neurosurg Psychiatry 90(8):939-944. https://doi.org/10.1136/jnnn-2018-320242

16. Lipton RB, Goadsby PJ, Smith J, Schaeffler BA, Biondi DM, Hirman J, Pederson S, Allan B, Cady R (2020) Efficacy and safety of eptinezumab in patients with chronic migraine. PROMISE-2. Neurology. 94(13):e1365-e1377. https://doi.org/10.1212/WNL.0000000000009169

17. Schwedt T, Reuter U, Tepper S, Ashina M, Kudrow D, Broessner G, Boudreau GP, McAllister P, Vu T, Zhang F, Cheng S, Picard H, Wen S, Kahn J, Klatt J, Mikol D (2018) Early onset of efficacy with erenumab in patients with episodic and chronic migraine. J Headache Pain. 19(1):92. https://doi.org/1 0.1186/s10194-018-0923-6

18. Silberstein SD, Dodick DW, Bigal ME, Yeung PP, Goadsby PJ, Blankenbiller T, Grozinski-Wolff M, Yang R, Ma Y, Aycardi E (2017) Fremanezumab for the preventive treatment of chronic migraine. N Engl J Med 377(22):2113-2122. https://doi.org/10.1056/NEJMoa1709038

19. VYEPTI (2020) package insert. Lundbeck Seattle BioPharmaceuticals, Inc. Bothell, WA

20. Lipton RB, Dodick DW, Ailani J, McGill L, Hirman J, Cady R (2021) Patientidentified most bothersome symptom in preventive migraine treatment with eptinezumab: a novel patient-centered outcome. Headache 61(5):766776. https://doi.org/10.1111/head.14120

21. Headache Classification Committee of the International Headache Society (IHS) (2013) The international classification of headache disorders, 3rd edition (beta version). Cephalalgia. 33(9):629-808. https://doi.org/10.1177/ 0333102413485658

22. Garcia-Martinez LF, Raport CJ, Ojala EW, Dutzar B, Anderson K, Stewart E, Kovacevich B, Baker B, Billgren J, Scalley-Kim M, Karasek C, Allison D, Latham JA (2020) Pharmacologic characterization of ALD403, a potent neutralizing humanized monoclonal antibody against the calcitonin gene-related peptide. JPET. 374(1):93-103. https://doi.org/10.1124/jpet.119.264671

23. Dodick DW, Gottschalk CH, Tepper SJ, Friedman DI, Cady R, Smith J et al (2020) Eptinezumab demonstrated migraine preventive efficacy on day 1 after dosing: closed testing analysis from PROMISE-1 and PROMISE-2. Headache. 60(S1):107-108

24. Baker B, Schaeffler B, Beliveau M, Rubets I, Pederson S, Trinh M, Smith J Latham J (2020) Population pharmacokinetic and exposure-response analysis of eptinezumab in the treatment of episodic and chronic migraine. Pharmacol Res Perspect 8(2):e00567. https://doi.org/10.1002/prp2.567

25. Dodick DW, Gottschalk C, Cady R, Hirman J, Smith J, Snapinn S (2020) Eptinezumab demonstrated efficacy in sustained prevention of episodic and chronic migraine beginning on day 1 after dosing. Headache 60(10):22202231. https://doi.org/10.1111/head.14007

26. Winner PK, McAllister P, Chakhava G, Ailani J, Ettrup A, Krog Josiassen M, Lindsten A, Mehta L, Cady R (2021) Effects of intravenous eptinezumab vs placebo on headache pain and most bothersome symptom when initiated during a migraine attack: a randomized clinical trial. JAMA. 325(23):23482356. https://doi.org/10.1001/jama.2021.7665

27. Dodick DW, Lipton RB, Silberstein S, Goadsby PJ, Biondi D, Hirman J, Cady R, Smith J (2019) Eptinezumab for prevention of chronic migraine: a randomized phase 2b clinical trial. Cephalalgia. 39(9):1075-1085. https://doi. org/10.1177/0333102419858355

28. Ashina M, Saper J, Cady R, Schaeffler B, Biondi DM, Hirman J et al (2020) Eptinezumab in episodic migraine: a randomized, double-blind, placebocontrolled study (PROMISE-1). Cephalalgia. 40(3):241-254. https://doi.org/1 $0.1177 / 0333102420905132$

29. Silberstein S, Diamond M, Hindiyeh NA, Biondi DM, Cady R, Hirman J, Allan B, Pederson S, Schaeffler B, Smith J (2020) Eptinezumab for the prevention of chronic migraine: efficacy and safety through 24 weeks of treatment in the phase 3 PROMISE-2 (prevention of migraine via intravenous ALD403 safety and efficacy-2) study. J Headache Pain. 21(1):120. https://doi.org/1 $0.1186 / s 10194-020-01186-3$ 
30. Serrano D, Lipton RB, Scher Al, Reed ML, Stewart WBF, Adams AM et al (2017) Fluctuations in episodic and chronic migraine status over the course of 1 year: implications for diagnosis, treatment and clinical trial design. J Headache Pain 18(1):101. https://doi.org/10.1186/s10194-017-0787-1

\section{Publisher's Note}

Springer Nature remains neutral with regard to jurisdictional claims in published maps and institutional affiliations.

Ready to submit your research? Choose BMC and benefit from:

- fast, convenient online submission

- thorough peer review by experienced researchers in your field

- rapid publication on acceptance

- support for research data, including large and complex data types

- gold Open Access which fosters wider collaboration and increased citations

- maximum visibility for your research: over $100 \mathrm{M}$ website views per year

At BMC, research is always in progress.

Learn more biomedcentral.com/submissions 